\title{
Circulative Narrative Generation Based on the Mutual Transformation between Narrative Conceptual Structures and Music in the Integrated Narrative Generation System
}

\author{
Taisuke Akimoto \\ Graduate School of Informatics and Engineering, The University of Electro-Communications, 1-5-1 Chofugaoka \\ Chofu, Tokyo 182-8585, Japan \\ E-mail:t8akimo@yahoo.co.jp \\ Takashi Ogata \\ Faculty of Software and Information Science, Iwate Prefectural University, 152-52 Sugo \\ Takizawa, Iwate 020-0693, Japan \\ E-mail:t-ogata@iwate-pu.ac.jp
}

\begin{abstract}
We have proposed a new framework of narrative generation which generates narratives through the mutual transformation according to various pathways between narrative (conceptual representation) and music. This paper will continuously incorporate this framework into the integrated narrative generation system which is a comprehensive architecture for our narrative generation study and develop an experimental system combined with the integrated system. This new framework aims to apply various musical methods for composition and variation to narrative generation as a way to connect different types of media. An experiment of the mutual generation between music and narrative will show various transformation pathways or routes between music and narrative.
\end{abstract}

Keywords: integrated narrative generation system, narrative generation, music generation, structural transformation

\section{Introduction}

Issues on the similarity or correspondence relation between narrative and music have been discussed in musicology. ${ }^{1,2,3}$ Overview, based on the studies, music itself cannot express concrete meaning as narratives. However, a narrative and a piece of music can commonly be composed of a sequence of events although a temporal sequence of events in narrative is different from a thematic sequence of events is music. We focus on the common structures.

The major purpose of our current research is developing an integrated narrative generation system to incorporate a variety of narrative generation mechanisms into an organic architecture. ${ }^{4}$ The system consists of the following three large units: 1) Story generation mechanism generates the content of a narrative with a hierarchical conceptual structure using such knowledge units as conceptual dictionaries and story contents knowledge base. 2) Discourse generation mechanism transforms a story structure into the other conceptual structures considering how to narrate the story. 3) Expression mechanism generates representation by surface media including natural language, music and visual image.

One of the design concepts of the system is circularity or cyclicity. Although the one meaning is the ability to repeatedly generate narratives in one generation session, another meaning is the generation ability based on the mutual transformation relationship between narrative conceptual structures and the surface media expression. The transformation to a narrative 
conceptual structure from a surface medium, for example, will be also possible in the latter sense.

Music has been the first main object or medium to accomplish the concept. We have been studying narrative generation methods through mutual transformation according to various pathways between narrative and music based on the development of some experimental systems. The first system was presented by Ref. 5 . And the most recent system presented by Ref. 6 included eight routes of the transformation between narrative and music using automatic composition and musical variation mechanisms. However, the system was an independent experimental system in which only the relationship of narrative conceptual structures and music was simply defined.

The goal in this paper on the basis of the research is to incorporate the above experimental mutual transformation mechanism into the integrated narrative generation system. Music in this mechanism will not be just the accompaniment for a narrative. We aim to use musical knowledge \& techniques and conceptual knowledge \& techniques in fusion by the medium of music. Mechanisms for story \& discourse and the musical expression mechanism are organically integrated together. This paper will introduce the current status of the development.

There are various systems that generate the background music for a narrative in the area of artificial intelligence (e.g., Ref. 7). However, although narrative generation systems or computational narrative models are currently an independent study genre, ${ }^{8}$ the other researches do not exist to treat music from the view point of narrative generation or the structural correspondence between narrative and music except for a series of our study.

\section{Basic Methods of Narrative and Musical Processing}

This section will present summaries of the integrated narrative generation system and the musical processing. Most of the foundational parts have been implemented with Common Lisp incrementally. The system is currently operating as a unified system.

\subsection{Narrative structures and the processing}

A story and a discourse generated in each of the mechanisms are respectively a same form of hierarchical tree structure. The basic units are an event or event concept and a relation. The former is a type of frame structure. And the latter connects two or more events, or two or more sub-structures with some events and one or more relations. An event concept is equivalent to a case structure with a verb concept and instances as values of some cases corresponded to the verb concept including agent, object, location, and so on. For example, "(event Eat (agent Boy) (object Apple) (location Park))" is the description of an event concept.

Both of structures of a story and a discourse are generated or transformed through the application of various types of techniques. We call the structural operational techniques associated with the story generation mechanism and the discourse mechanism "story techniques" and "discourse techniques" respectively. The name of "narrative techniques" is a collective term which contains story techniques, discourse techniques and moreover techniques for the generation by the surface expression media. Basically, for each of the story techniques and discourse techniques, the input (or target) is an event or a substructure with some events and the output is a newly expanded or transformed structure. The difference is the point that story techniques basically add a new element to the existing tree structure to expand it, while discourse ones transform a tree structure to another structure including the same event elements. A variety of types of narrative techniques can be integrated grounded on this common principle. The current version of the system has about thirteen types of story techniques and similarly ten types of discourse techniques.

\subsection{Musical structures and the processing}

The mutual relationship between a story structure and a discourse structure in the level of conceptual narrative are corresponded to the mutual relationship between a piece of "original music" and the "variation music" in the level of music. The basic method of the mechanism is to relate a story structure and a discourse structure to the structure of a piece of original music and a piece of variation music according to the concrete procedure explained in the following part. The mutual transformation is processed based on relational mappings.

The tree structure of a story is mapped to a musical tree structure with the similar form. Fig. 1 shows the structure of a piece of original music. Each of the 
internal nodes in the musical tree is equal to "primarysecondary" or "secondary-primary" relation between the child nodes. The primary side is more important musical part than the secondary side. Each of the leaf nodes, on the other hand, is corresponded to a musical event. A musical event is equal to a set of the following musical elements: one or more motifs, the harmony, and the duration defined in the number of quarter notes from 1 to 16. The musical events at the leaf nodes are sequentially played in original music. A motif is a basic musical unit consisting of a sequence of notes and has the same duration with the musical event. Each of the notes has a pitch and its duration. Each of the musical events is corresponded to an event in the story and each of the motifs is corresponded to one of the instances in the story.

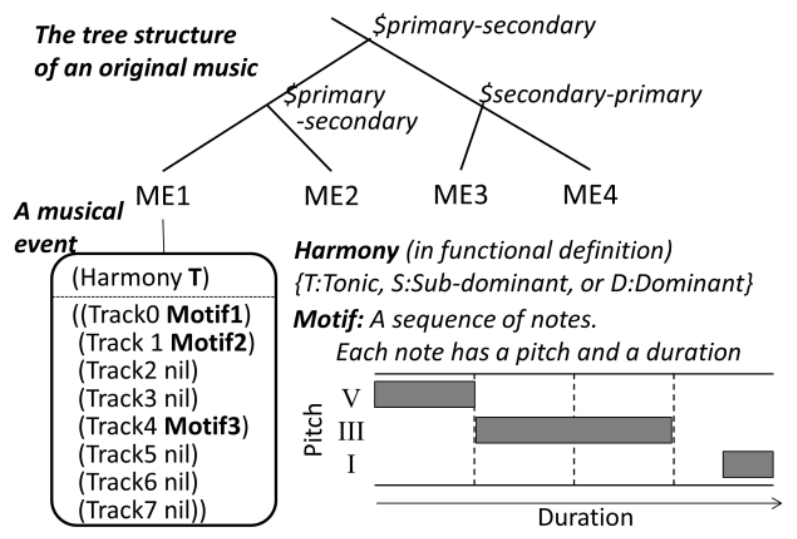

Fig. 1. The structure of a piece of original music.

A piece of original music is generated through the operation of the structure and elements. The procedure of the original music generation from a story is as follows: 1) The mechanism generates motifs associated with each instance in the story. Each of the motifs is made with a sequence of notes which are selected at random. 2) It transforms each of the internal nodes, namely relations, in the story into the musical internal node using a set of rules for associating the two groups of internal nodes. 3) It decides a harmony in each musical event using a set of rules for deciding the harmony progression with a musical tree structure. 4) It transforms each event in the story into a musical event by substituting each instance to the corresponding motif.

On the other hand, the structure of a piece of variation music is also represented as a tree structure form which is transformed from the structure of a piece of original music using variation techniques. Each of the variation techniques is defined as a manipulation of the musical tree structure or the element. There are such techniques as the move and deletion of a sub-structure of a musical tree, the addition of a musical event created newly into a musical tree, and the modification of musical elements like tempo, pitch and volume.

\section{A Mutual Transformation Mechanism between Narrative and Music}

\subsection{The system configuration}

We show in Fig. 2 the macro framework of the integrated narrative generation system including musical mechanisms. The musical mechanisms consist of an original music mechanism and a variation music mechanism. The former generates a piece of original music from a story, a piece of variation music, or a piece of original music. The latter, on the other hand, generates a piece of variation music according to a piece of original music, a piece of variation music, or a discourse structure. We have incorporated in addition to the above a mechanism for generating a discourse structure from a piece of variation music into the discourse mechanism. A mechanism to generate a story structure from a piece of original music is currently under development.

The user initially inputs generative parameters for directing the story generation. The following four parameters are prepared: "macro-structure", "length", "unreality" and "repetition". The user continuously sets the generation pathways from the next prescribed sequences: "S-D-NL", "S-D-NL-I", "S-D-VM" and "SOM-VM-D-NL". Each of the S, D, NL, I, OM and VM mean Story, Discourse, Natural Language, Image, Original Music and Variation Music respectively.

The story mechanism is always executed firstly to generate a story by using adequate story techniques according to the generative parameters for story. Then the method sends the result by the previously executed mechanism to the next mechanism.

The user is necessary to input the following information in the middle of a continual generation process. When the discourse mechanism is executed, the user needs to set the generative parameters and the other settings for discourse generation. The user needs to select one or more variation techniques to be used for the musical variation processing. For generating a new 


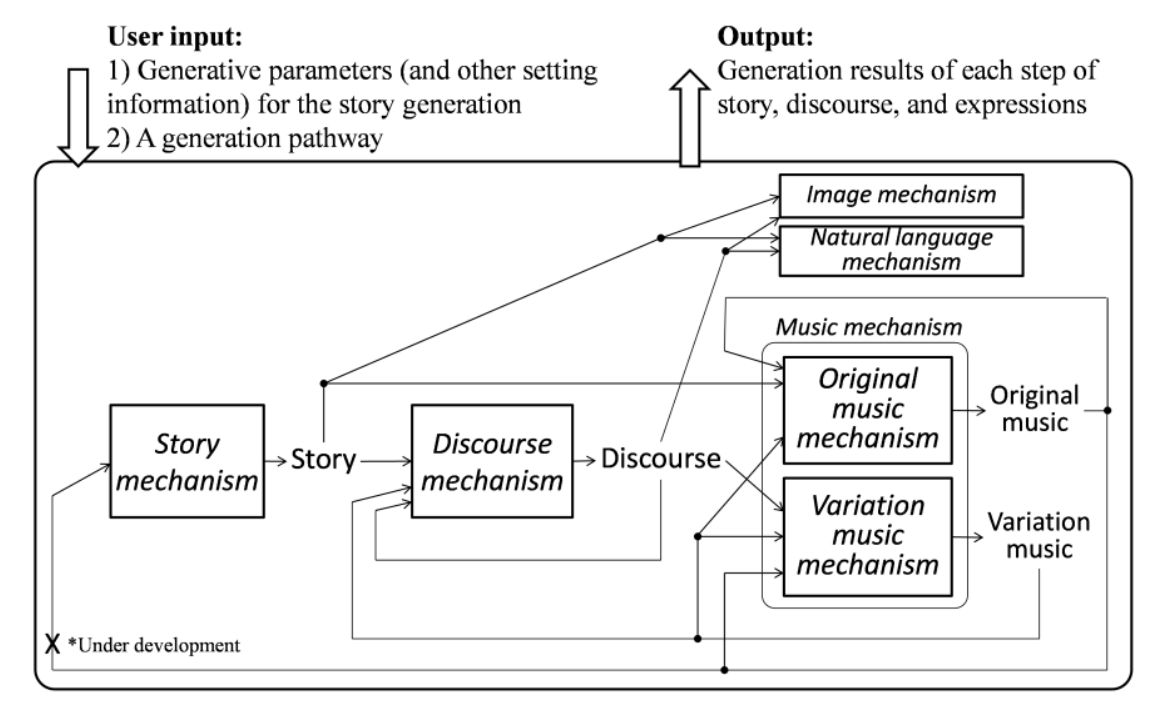

Fig.2. The expanded framework by musical mechanisms of the integrated narrative generation system.

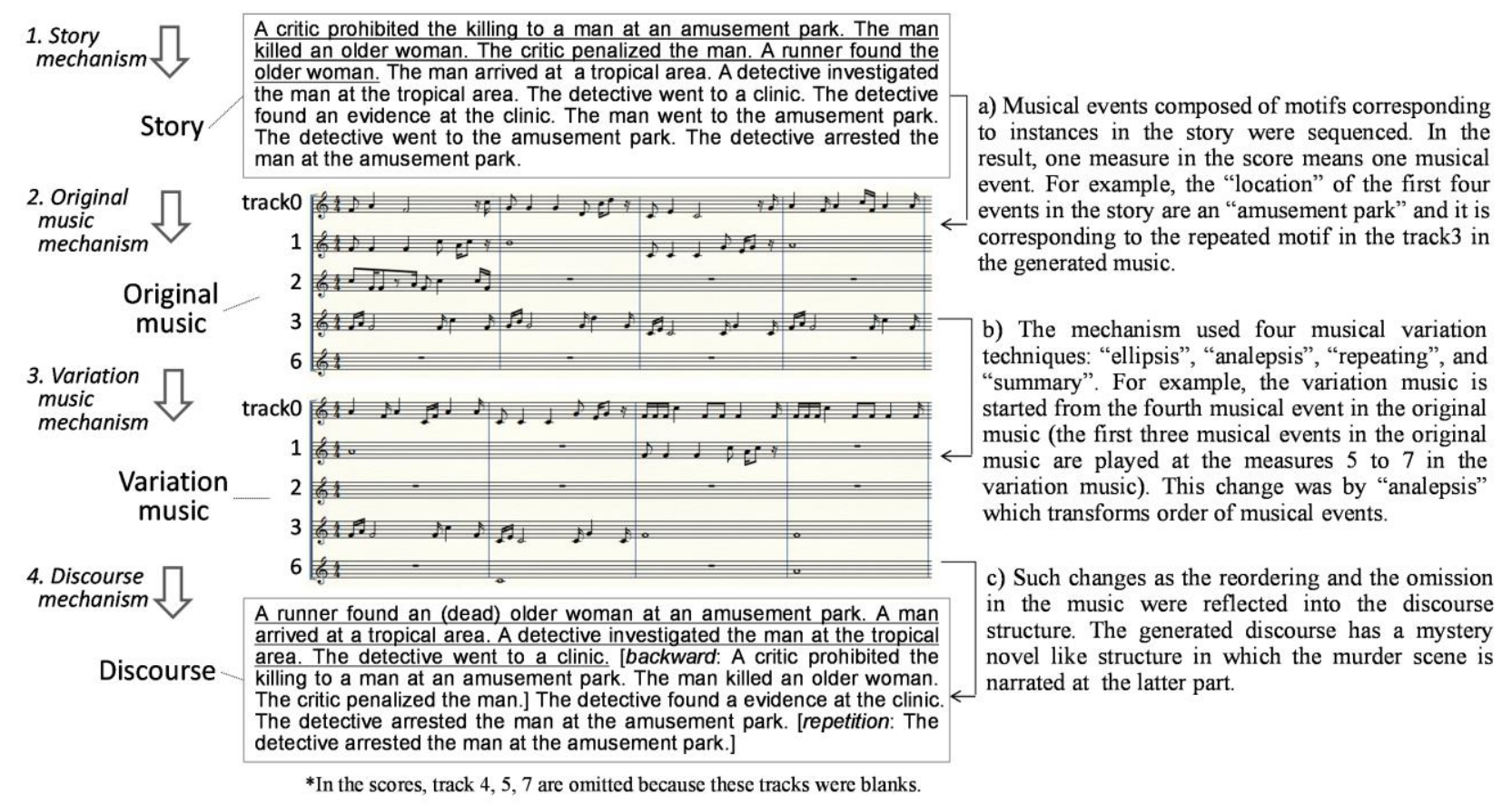

Fig.3. A generation flow example.

piece of original music from an existing piece of original music, the user is required to select one or more operational techniques to change the original music structure.

\subsection{The example of a generation flow}

We will present the example of a narrative generation process including music generation by the method described above. The initial values inputted by the user are generative parameters such as "(macro-structure 2) (length 3) (unreality 1) (repetition 2)" and the generation pathway is set as "S-OM-VM-D-NL".

The result of each generation phase is shown in Fig. 3 in order. The surface representation by language of story and discourse in the figure is English translation by hand from Japanese language texts generated by a 
simple language generation mechanism in the integrated narrative generation system. These language texts are transformed from each of the conceptual representation forms. On the other hand, only first four musical events and the scores by hand are shown in the results of original music and variation music.

Conceptual processing and musical processing in a series of generation are mutually connected as shown in the example. To add the function of story generation from a piece of original music, which is not implemented in the current version, will contribute more free and flexible generation since we will be able to start the system's generation process from a piece of original music. It means the narrative generation from music in the extreme sense.

One of the issues to be solved in the current implementation is that the current discourse mechanism cannot transform musical structures into the corresponding discourse structure in several types of musical variation techniques, namely the modification techniques for such musical elements as tempo, pitch, and volume because of the theoretical problem on structure transformation considering the meaning in narrative and music.

\section{Conclusion}

This paper has presented the expanded framework of the integrated narrative generation system through the experimental system implementation. The important point of this framework is that musical generation and variation techniques are incorporated into the system to enable the mutual transformation between music and narrative. The previous version of the integrated narrative generation system basically generated a story structure, a discourse structure and expression representations including music in order, while introducing the mutual transformation mechanisms enabled the system to execute a variety of generation routes. Actually, we showed a generation example of the mutual transformation in which a piece of music is generated from a narrative structure and a musical variation of the piece causes a change of the narrative structure. We would like to verify the abilities in the several sides of the mutual transformation mechanisms between narrative and music after the completion.

The program of narrative structure processing has been implemented based on relatively sound theoretical foundations by a kind of knowledge-based architecture with narrative techniques and conceptual dictionaries. The background of the musical composition and variation, on the other hand, has been weak in both sides of theory and design. Thus, one of the next major topics is introducing musical theories and techniques into the part of the musical processing.

\section{References}

1. R. Walsh, The common basis of narrative and music, Storyworlds: A Journal of Narrative Studies, 3(1) (2011) 49-71.

2. F. E. Maus, Music as narrative, Indiana Theory Review, 12 (1991) 1-34.

3. L. Kramer, Musical narratology: A theoretical outline, Indiana Theory Review, 12 (1991) 141-162.

4. T. Akimoto and T. Ogata, Macro structure and basic methods in the integrated narrative generation system by introducing narratological knowledge, in Proc. of 11th IEEE International Conference on Cognitive Informatics \& Cognitive Computing (Kyoto, 2012), pp. 253-262.

5. F. Kobayashi and T. Ogata T, Narrative and music as variation: Transformation of musical structure based on narrative discourse theory, in Proc. of the 9th International Symposium on Artificial Life and Robotics, 1 (Oita, 2004), pp. 170-173.

6. T. Akimoto, J. Endo and T. Ogata, The expansion of paths in the mutual transformation mechanism of music and narrative, in Proc. of the 11th IEEE International Conference on Cognitive Informatics \& Cognitive Computing (Kyoto, 2012), pp. 230-239.

7. M. O. Jewell, M. X. Nixon and A. Prügel-Bennett, Statebased sequencing: Directing the evolution of music, in Proc. of the International Computer Music Conference (Barcelona, 2005).

8. P. Gervás, B. Lönneker-Rodman, J. C. Meister and F. Peinado, Narrative models: Narratology meets artificial intelligence, in Proc. of Satellite Workshop: Toward Computational Models of Literary Analysis, 5th International Conference on Language Resources and Evaluation (Genova, 2006), pp. 44-51. 